\title{
Palmprint Recognition Based on Deep Convolutional Neural Networks
}

\author{
Xueqiu Dong ${ }^{1, \text { a, }}$, Liye Mei ${ }^{1, ~ b}$, and Junhua Zhang ${ }^{2, ~ c ~}$ \\ ${ }^{1}$ School of Information Science and Engineering, Yunnan University, Kunming, 650091, China \\ a876237391@qq.com, ${ }^{b}$ liyemei@mail.ynu.edu.cn, ${ }^{c}$ jhzhang@ynu.edu.cn \\ *Corresponding author
}

Keywords: palmprint recognition, feature extraction, convolutional neural network

\begin{abstract}
Palmprint recognition, as one of the current biometric technologies, has received extensive attention and research. There are difficulties in the traditional artificially defined feature when it extracts problems. In order to identify palmprints simply and efficiently, meanwhile, to eliminate the problem of coupling palmprint recognition with manually defined feature extraction problems, an algorithm for palmprint recognition directly on palmprints is proposed. The ability of automatic feature extraction utilizing convolution network is used to learn the palmprint features from the palmprint database, and the network can adapt to the variety of the palmprint by the design of the training data. The experimental results show that the palmprint recognition approach based on the convolution neural network (CNN) achieves an overall accuracy of 99.95\%, and the model parameters are reduced by a lot compared with those in the standard network model. Its accuracy is much better than that of traditional palmprint recognition algorithm.
\end{abstract}

\section{Introduction}

In modern society, people have higher requirements for the accuracy, practicability and security of identity authentication. Traditional identity authentication has the disadvantages of being easily lost and misappropriated. The biometric recognition technology provides a great help for solving problems by distinguishing their physiological and behavioral characteristics. Palmprint recognition is a biometric recognition technology proposed in recent years, such as face recognition [1], fingerprint recognition [2], palmprint recognition, iris recognition [3] and vein recognition [4].

We propose a palmprint recognition algorithm based on a deep convolutional neural network (CNN) that uses the ability of the network to automatically extract palmprint convolution features and learns the characteristics of palmprint from large amounts of data. Meanwhile, text recognition can be performed directly on the master map of palmprints. The experimental results show that the proposed method can really improve the accuracy of the test. In this paper, first, we introduce the effect of the recognition of the network and other methods used in this paper. Then,we prepare the data required for the algorithm and design the network, and adjust the parameters of the network according to the training of the data so that they can extract features with distinctiveness. The trained features will be predicted with the new palmprint. In addition, in order to improve the accuracy of the algorithm, we normalize the data, the normalization can improve and it may also 
improve the accuracy of the calculation. Finally, the experiments prove that the algorithm can obtain good results. The algorithm fully mines the information in the palmprint image and does not require any artificially defined feature extraction steps, which greatly simplifies the difficulty of the palmprint recognition algorithm.

\section{Related Work}

With the development of deep learning, convolution neural network (CNN) exert its potential. It has been widely used in the fields of image, phonetics, natural language understanding, etc. Since the network itself has automatic feature extraction, it does not need to rely on artificially defined features. Looking at the current general trend of development, we want to get higher accuracy through deeper and more complex networks, but this kind of network has its shortcomings in model size and running speed. In the establishment of a small and effective neural network, there are two kinds compressed pre-training models and direct training of small networks, the main reason is to optimize the delay and take into account the model size.

Recently, several research have studied palmprint identification based on deep learning approaches. In [5], to further improve the performance of multispectral palmprint recognition method, propose a novel method based on MPELM. Firstly, all palmprint images are preprocessed by David Zhang method. Then, use image fusion method based on fast digital shearlet transform to fuse the multispectral palmprint images. At last, use the proposed MPELM classifier to determine the final multispectral palmprint classification. The experimental results showed that the proposed model achieved an average accuracy of 97.33\%. In [6], a novel Gabor-based kernel principal component analysis (PCA) method by integrating the Gabor wavelet representation of palm images and the kernel PCA method for palmprint recognition is proposed. The results demonstrated that the recognition accuracy of the proposal was up to $95.17 \%$. In [7], an automated scanner-based palmprint recognition system is proposed. The system automatically captures and aligns the palmprint images for further processing. Several linear subspace projection techniques have been tested and compared like fisher discriminant analysis (FDA) and independent component analysis (ICA). The results demonstrated that the recognition accuracy of the proposal was up to 95.2\%, 95.7\%. In [8], X. Xu and Z. Guo presents a method of representing the multispectral palmprint images by quaternion and extracting features using the quaternion principal components analysis (QPCA) to achieve better performance in recognition. QPCA is used to extract features of multispectral palmprint images. The dissimilarity between two palmprint images is measured by the Euclidean distance. The experiment shows that given 3000 testing samples from 500 palms, the best GAR is $98.13 \%$. In [9], a novel contactless Palmprintrecognition system using palm print principal line-based feature extraction technique has been proposed. Distances from endpoints to endpoints and point of interception to endpoints were calculated and transformed to frequency domain by the application of Discrete Fourier Transformed (DFT) technique. The proposed model was trained and achieved an accuracy of $95.48 \%$. In [10], an algorithm to do so that works with discrete wavelet transforms. The results demonstrated that the recognition accuracy of the proposal was up to $98 \%$. The results show that the convolution neural network has been widely used in palmprint recognition and achieved good results. So far, the MobileNet based model has not been applied to palmprint recognition. This paper presents a new CNN model for palm print recognition.

\section{Palmprint Recognition Method}

Convolution neural network (CNN), as one of the most successful deep learning models applied in the field of image recognition, discriminative pattern features can be automatically learned from a large amount of data. When the amount of data used is relatively large, it can achieve the effect of 
close to human eye recognition. Therefore, we apply it to palmprint recognition and get a good recognition rate by adjusting the network structure. The network structure is shown in table 1 .

\subsection{Data Preparation}

Data comes from palm database of Hong Kong University of science and technology [13]. PolyU palmprint database is considered to be one of the most authoritative public palmprint database. It contains 6,000 grayscale images from 500 different palms, each of which has 128x128 pixels, 5,400 as a training set and 600 as a test set. In order to reduce the over-fitting in the training stage, slight distortion was introduced in the experimental stage. Meanwhile, for the sake of increase the speed of the solution, the accuracy of the calculation can also be improved. At the experimental stage, we performed normalization of the mean variance of the data. One cannot directly normalize the test data set by the formula, but use the mean and variance of the training data set to normalize the test data set.

\subsection{Building Network}

MobileNet is the basic unit of deep separable convolution, deep separable convolution can be decomposed into two smaller operations: depthwise convolution (DC) and pointwise convolution (PC)[15]. Depthwise convolution uses different convolution kernels for each input channel, equivalent to a convolution kernel corresponding to an input channel. In fact, pointwise convolution is a standard convolution and a $1 \times 1$ convolution kernel. The depthwise separable convolution (DSC) applies depthwise convolution of convolution to each different input channel, then pointwise convolution is applied to combine the output of the above again, so the overall effect is similar to a standard convolution, but it will greatly reduce the amount of calculation and model parameters. The network adopts a form of factorized depthwise separable convolution, which can not only reduce the computational complexity of the model, but also greatly reduce the size of the model.

MobileNet has 28 layers. The first layer is a full convolution (3x3) which need not deptwise separable convolution. All layers are followed by a batchnorm [14] and ReLU nonlinearity with the exception of the final fully connected layer which has no nonlinearity and feeds into a softmax layer for classification. the downsampling part is achieved through the first layer convolution and part of depthwise convolution's stride equal 2. Some of MobileNet's architecture is shown in fig 1[15].

Inspired by the classical MobileNet their performance, a deep convolutional neural network model is proposed to identify palmprint. Firstof all, a network structure is designed, which is based on the standard MobileNet model. For the perception of the convolution kerne a larger sized the convolution kernel has a stronger ability to extract the macro information of the image, and vice versa. The network input layer 1 is standard convolutional layer. The second convolutional layer is deptwise convolution. The third convolutional layer is pointwise layers. The fourth is convolutional layer is deptwise convolution. The fifth convolutional layer is pointwise layers. The sixth is convolutional layer is deptwise convolution. Theseventh convolutional layer is pointwise layers. The eighth is convolutional layer is deptwise convolution. The ninth convolutional layer is Pointwise layers. The tenth is convolutional layer is deptwise convolution. The eleventh convolutional layer is pointwise layers and is then followed by a FC layer with a size of $1024 \times 500$ and the final layer is a Softmax layer. All its layers are batchnorm [10] and ReLU non-linear, only the final full-connected layer has no nonlinearity and is fed into the softmax layer for classification. The correlation model parameters are shown in table 1. 

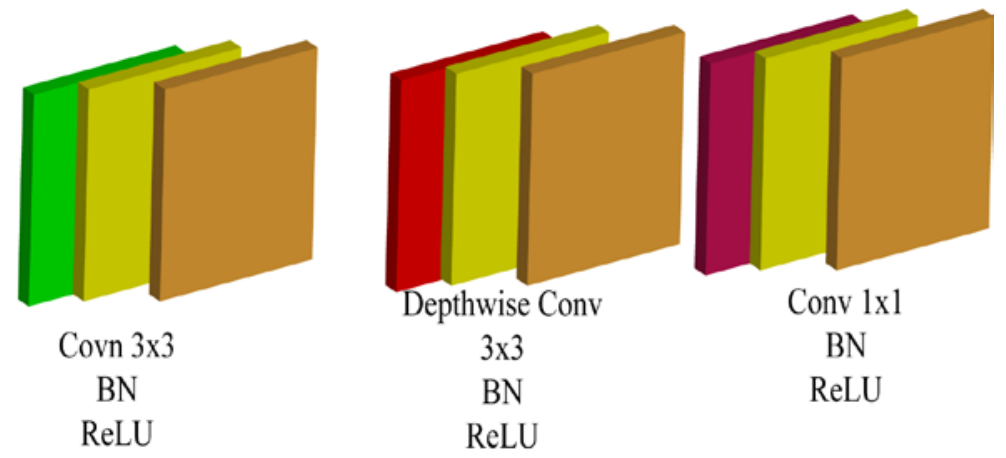

Fig. 1 Left: Standard convolutional layer with batchnorm and ReLU. Middle and right: Depthwise Separable convolutions with Depthwise and Pointwise layers followed by batchnorm and ReLU.

Table 1. MobileNet Body Architecture

\begin{tabular}{ccc}
\hline Type/Stride & Filter Shape & Input Size \\
\hline Conv / s2 & $3 \times 3 \times 3 \times 32$ & $224 \times 224 \times 3$ \\
Conv dw/s1 & $3 \times 3 \times 32 \mathrm{dw}$ & $112 \times 112 \times 32$ \\
Conv / s1 & $1 \times 1 \times 32 \times 64$ & $112 \times 112 \times 32$ \\
Conv dw / s2 & $3 \times 3 \times 64 \mathrm{dw}$ & $112 \times 112 \times 64$ \\
Conv/s1 & $1 \times 1 \times 64 \times 128$ & $56 \times 56 \times 64$ \\
Conv dw / s1 & $3 \times 3 \times 128 \mathrm{dw}$ & $56 \times 56 \times 128$ \\
Conv / s1 & $1 \times 1 \times 128 \times 128$ & $56 \times 56 \times 128$ \\
Conv dw / s2 & $3 \times 3 \times 128 \mathrm{dw}$ & $56 \times 56 \times 128$ \\
Conv / s1 & $1 \times 1 \times 128 \times 256$ & $28 \times 28 \times 128$ \\
Conv dw / s1 & $3 \times 3 \times 256 \mathrm{dw}$ & $28 \times 28 \times 256$ \\
Conv / s1 & $1 \times 1 \times 256 \times 256$ & $28 \times 28 \times 256$ \\
FC / s1 & $1024 \times 500$ & $1 \times 1 \times 1024$ \\
Softmax / s1 & Classifier & $1 \times 1 \times 500$ \\
\hline
\end{tabular}

\subsection{Training Details}

We train the network on the popular deep learning platform Keras[11], the objective function is defined as the softmax loss function and batch size is set to 20. Each convolution layer uses the "Gaussian" method to initialize the weights and the bias is set to 0 . The basic learning rate for each layer is set to 0.001 . We use the SGD algorithm, which the weight decay and momentum are set separately $0.0005,0.9$, to optimize parameters, the Train with rmsprop[12]. For every 7 epochs training, the basic learning rate will be halved and the training model could be obtained through 100 epochs training.

\section{Experiment Results}

In this experiment, palmprint data applied from palmprint database of Hong Kong University of science and technology [13]. Available from http://www.comp.polyu.edu.hk/ biometrics. The experiment was performed in an environment with Intel(R) Core(TM) i7-7820HK 2.90GHz processor, 32.0GB memory, 64-bit operating system and Python 3.6. To verify the validity of our algorithm, in this section, we simply describe the palmprint database and compare with the research results of other methods. The network was constructed and optimized by the deep learning framework Keras. Its network structure is shown in table 1. The random gradient descent method 
was used to optimize the network parameters. After 70 minutes of training, a good effect was achieved.

\subsection{Experiment Database}

We make use of the Hong Kong Polytechnic University (PolyU) palmprint database, this database includes 6000 palmprint images from 500 individuals with 6 palmprint images from each.There are $12 \times 500=6000$ grayscale palmprint images in our database. The size of each palmprint is $128 \times 128$ pixels, as shown in fig 2 .
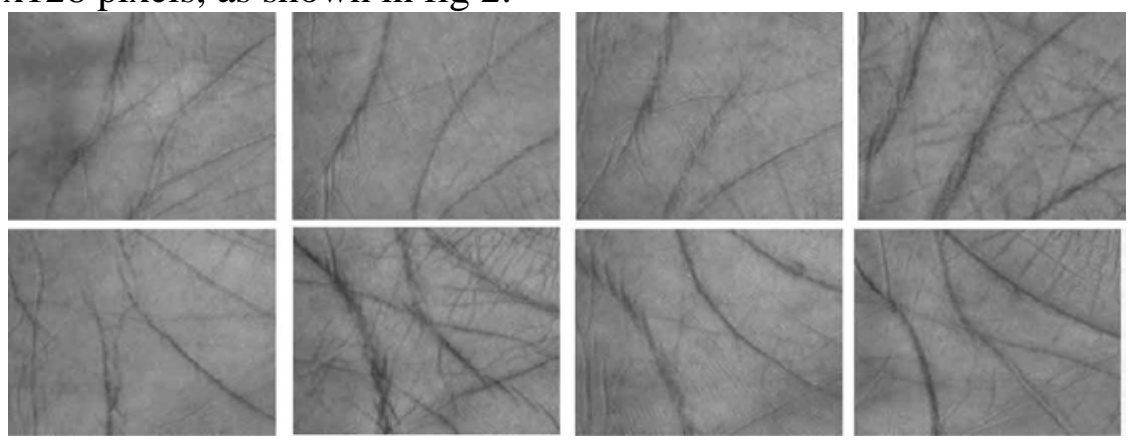

Fig. 2 PolyU palmprint image

\subsection{Experiment Results}

In the experiment, we used 5,400 palmprint grayscale images as training data and then used 600 palmprint grayscale images as test data. After several rounds of training, the correct recognition rates obtained were 99.95\%, 99.95\%, respectively. Because palmprints are abundant features wih it may be affected by the resolution, which may also lead to recognition errors. Comparisons with other methods are shown in table 2.

Table 2 comparison with other methods

\begin{tabular}{cc}
\hline Methods & Recognition Rate \\
\hline PCA + GWR & $95.17 \%$ \\
QPCA & $98.13 \%$ \\
MPELM & $97.33 \%$ \\
FDA & $95.2 \%$ \\
ICA & $95.7 \%$ \\
DFT & $95.48 \%$ \\
DWT & $98 \%$ \\
Our work & $\mathbf{9 9 . 9 5 \%}$ \\
\hline
\end{tabular}

We can see that our algorithm exceeds most classical algorithms from table 2. Although the accuracy of our algorithm is lower than the literature [16], the literature [16] proposes a hybrid feature of palmprint recognition, this hybrid feature is a combination of two features of the palm line feature and the wavelet feature, extract palm lines by applying Laplacian masks on smooth images and the wavelet transform is applied to three stages, derive wavelet features from local wavelet of Image, and then using the minimum distance classifier to performe model matching. This algorithm is easily interfered by resolution and noise. Our algorithm does not rely on any preprocessing and feature extraction, and is a feature learned from a large amount of palmprint data. Therefore, can adapt to a lot of data and to get high accuracy.

In addition, we explore the relationship between experimental results and training data volume. 
Fig3 shows the relationship between the accuracy of the MobileNet network and the number of iterations during the training process for different training data volumes. With the number of iterations increase, the accuracy rate of the verification set under different amounts of iterations volumes rises first and finally stabilizes.

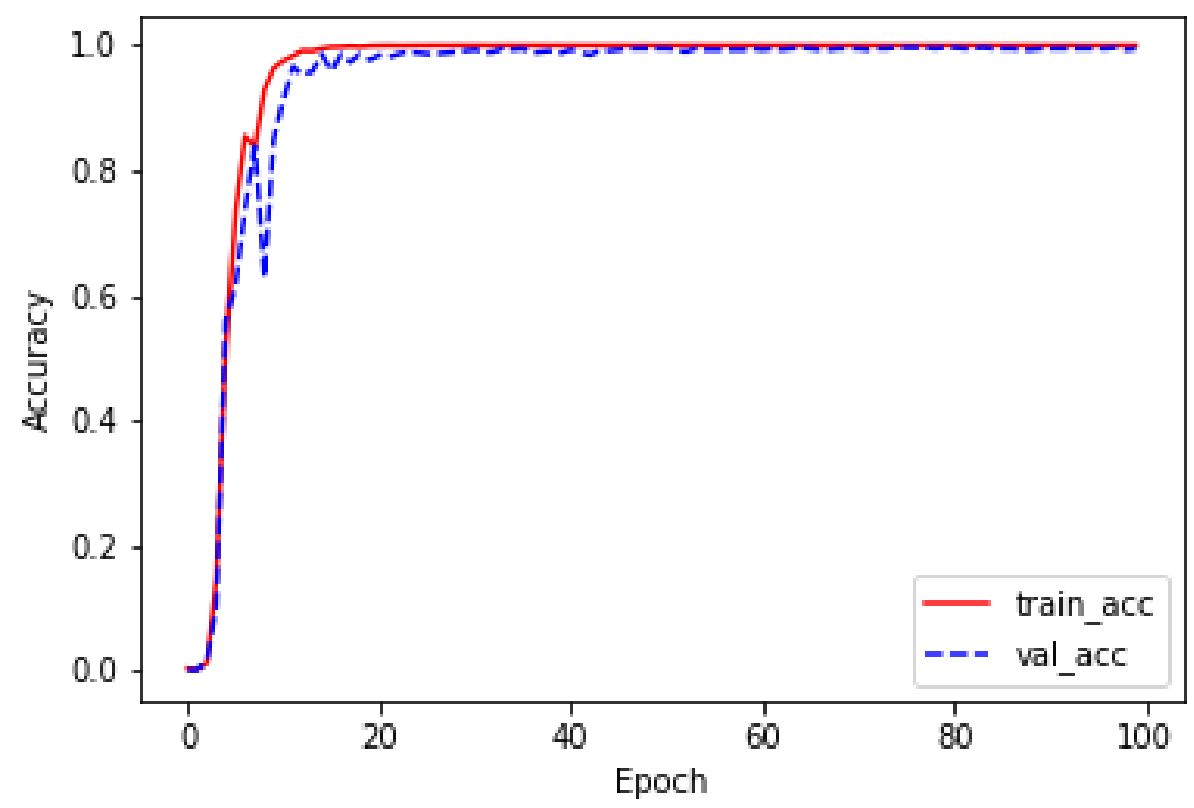

Fig. 3 The accuracy of training data and test data with different amounts of iterations

\section{Conclusion}

In this paper, we propose a palmprint recognition algorithm based on Convolutional Neural Networks (CNN), palmprint recognition method which can directly act on grayscale image, the ability of using network automatic feature extraction to learn palmprint database and to get the palmprint features, through the design of training data, the network can adapt to the diversity of palmprint. Through the effective design of network and training data, the palmprint feature can be extracted from the palmprint original without image preprocessing and other special extraction, thus to effective identification. The experimental results show that the method is efficient and reliable in the recognition of palmprint, and obtains higher accuracy.

\section{Acknowledgment}

This work was financially supported by the National Natural Science Foundation of China (No.61361010).

\section{References}

[1] S. Z.Gilani, A.Mian. Towards Large-Scale 3D Face Recognition// International Conference on Digital Image Computing: Techniques and Applications. IEEE, (2016) 1-8.

[2] A. K. Jain, A.A. Ross, K. Nandakumar. Fingerprint Recognition// Handbook of Biometrics. Springer US, (2008) 1-21.

[3] R.P. Wildes. Iris recognition: an emerging biometric technology. Proceedings of the IEEE, 1997, 85(9):1348-1363.

[4] A.H. Choi, J. R .Agre, D. T.Toledano, et al. Vein Recognition// Encyclopedia of Biometrics. (2009)

[5] X. Xu, L. Lu, X. Zhang, H. Lu, and W. Deng, "Multispectral palmprint recognition using multiclass projection 
extreme learning machine and digital shearlet transform,” Neural Computing and Applications, vol. 27, no. 1, 2016, pp. 143-153.

[6] M. Ekinci and M. Aykut, “Gabor-based kernel PCA for palmprint recognition,” Electronics Letters, vol. 43, no. 20, 2007, pp. 1077-1079.

[7] Tee Connie, Andrew Teoh Beng Jin, Michael Goh Kah Ong, David Ngo Chek Ling, “An automated palmprint recognition system”, Image and Vision Computing, Vol. 23,2005, pp.501-515.

[8] X. Xu and Z. Guo, “Multispectral palmprint recognition using quaternion principal component analysis,” IEEE Workshop on Emerging Techniques and Challenges for Hand-Based Biometrics, 2010, pp. 15.

[9] M. O. Rotinwa-Akinbile, A.M. Aibinu1 and M. J. E. Salami, "Palmprint Recognition Using Principal Lines Characterization”, First International Conference on Informatics and Computational Intelligence,2011, pp. 278 - 282.

[10] H.B. Kekr , K .Tanuja Sarode, A.A.Tirodkar, "A study of the efficacy of using Wavelet Transforms for Palm Print Recognition”, International Conference on Computing, Communication and Applications (ICCCA),2012, pp. 1-6.

[11] Information on https://keras.io/getting-started/faq/\#how-can-i-save-a-keras-model

[12] T.Tieleman and G. Hinton. Lecture 6.5-rmsprop: Divide the gradient by a running average of its recent magnitude. COURSERA: Neural Networks for Machine Learning, 4(2), 2012. 4 [34] M. Wang.

[13] UST Hand Image database[DB /OL].http: / /visgraph.CS.,ust.hk / biometrics /Visgraph—web /indef.html.

[14] S.Ioffe and C. Szegedy. Batch normalization: Accelerating deep network training by reducing internal covariate shift.arXiv preprint arXiv: 1502.03167. (2015)

[15] A. G.Howard, M. Zhu, B.Chen, et al. MobileNets: Efficient Convolutional Neural Networks for Mobile Vision Applications (2017)

[16] S. A.Mistani, S.Minaee, E. Fatemizadeh . Multispectral Palmprint Recognition Using a Hybrid Feature. Path, 2015, 10: págs. 109-136. 